\title{
A New Definition of Body Schema with Respect to Body-Centered Vs External Frames of Reference
}

\section{Rodrigues MRM}

Physical Therapist, Faculty Lecturer, School of Physical and Occupational Therapy, McGill University, Montreal, Canada

*Corresponding Author: Marcos R. M. Rodrigues, PT, MSc, School of Physical and Occupational Therapy, McGill University, Montreal, Quebec, Canada.
Received: September 28, 2020

Published: October 28, 2020

(C) All rights are reserved by Rodrigues

MRM.

\begin{abstract}
Moving is a way of experiencing the world and the way we move, as well as the way we perceive the environment around us, is relevant to this experience. The world we live in, however, is more than an ensemble of coordinates and the body is more than a passive machine ruled by laws of mechanics. A pragmatic view arised in 1920, when Held implied that body schema plays an important role in the control of action, involving aspects of both central and peripheral nervous systems. This is typically a non-conscious process and is used primarily for spatial organization of action.

The representation of one's own body is quite different from any other type of representation. It arises from the continuous and constant update of bottom-up and top-down information, both from internal and external bodily inputs. These inputs encompass different sources of information (e.g. visual, proprioceptive, interoception, nociception, motor behavior, etc.) and their interaction allows for building up body representations. Due to the complexity of such representation, it is widely accepted that there is more than one single body representation and many studies have tried to functionally fractionate different body representations, although, up to now, there is not a universally accepted taxonomy [1]. The identification of components of body representation is a challenge and the main interest in studying the egocentric frame of reference relies on the necessity of better understanding the first-person experience [2].

The lack of a proper definition of body schema has been leading to enduring methodological and conceptual misunderstandings in various fields. In rehabilitation, this lack of definition, as well as the unclear contribution of the concept of body schema in the context of the different frames of reference, ego- and exocentric, create a gap in the translation of this knowledge in the context of improving patient care.

Establishing a definition of body schema is still very challenging since there is not definitive evidence about the multiple factors directly influencing it, however a new definition might shed a light on this topic for it considers the contribution of both internal and external factors: Body schema is a spatiotemporal, continuously updated, modular and coherent representation of the physical body in the ventral nervous system, integrating a multitude of sensorimotor processes, serving as a guide for movements to be performed as accurately as possible while adapting to the constraints of the environment.
\end{abstract}

Keywords: Body Schema; Frames of Reference; Egocentric; Body-centered; Exocentric; Allocentric

\section{Abbreviations}

CNS: Central Nervous System; PPC: Posterior Parietal Cortex

\section{Background}

Living creatures, in order to move, confront a physical reality that has shaped their architecture as autonomous mobile entities 
because they are equipped with instruments adapted to read the spatial relationships between elements that are discernible by their sense organs [3]. Moving is a way of experiencing the world and the way we move, as well as the way we perceive the environment around us, is relevant to this experience. The world we live in, however, is more than an ensemble of coordinates and the body is more than a passive machine ruled by laws of mechanics [4]. Both are dynamically embedded systems that feed each other in order to promote inclusiveness and an optimal spatial navigation throughout the world and its re-entrances, thus requiring the integration of multisensory information relating to the body in space [5].

For more than a century, sensory and motor maps have been identified in the cerebral cortex, these maps being mirror images of the physical arrangement in the body of the sensory inputs or the motor outputs $[6,7]$. Long before that, René Descartes (1596 1650) was one of the first philosophers to shed light on the subject by referring to a differentiation between mind and body [8]. Descartes pointed out that thinking itself does not depend on any material thing in order to exist, leading to the conclusion that thoughts belong to a non-spatial substance that is distinct from matter. However, Descartes did not account for the fact that he, as a thinking thing, is in fact a complex material system. He also stated that "a mind cannot be understood to be shaped or in motion, nor can a body understand or sense anything" (ibid, Second Meditation, Volume 7, page 28) and human beings, however, are supposed to be combinations of mind and body so that that the choices of the mind can cause modes of motion in the body, and motions in certain bodily organs cause modes of sensation in the mind.

Although the discussions on the internal spatial representations of the body were first conceptualised by Münk (1890) and Bonnier (1905) [5], the discussion of the mind-body distinction started to receive input from a neurological perspective in 1911 when two different types of body representation were then proposed, one devoted to the appreciation of posture or passive movement and the other to the localization of body parts. At this time, Henry Head, a neurologist who pioneered the work on the somatosensory system and sensory nerves, along with the neurologist Gordon Morgan Holmes, first used the term "postural schema" to describe the disrupted spatial representation of patients after parietal lobe damage. Head and Holmes discussed two schemas (or schemata): one body schema for the representation of posture or movement and another body schema for the localization of stimuli on the body surface. "Body schema" became the term used for the organized models of ourselves [9]. This definition by Head and Holmes lasted for almost a century until a better understanding of neuroscience and the working of the brain emerged. This gradually led to a clearer differentiation of body schema from body image. Historically, the concepts of body image and body schema were used interchangeably. In order to avoid misuse and confusion, efforts to establish a proper definition have been made without a clear definition until today.

In 1920, Held implied that body schema plays an important role in the control of action, involving aspects of both central and peripheral nervous systems. This is typically a non-conscious process and is used primarily for spatial organization of action. It is therefore a pragmatic representation of the spatial properties of the body, which includes the length of limbs and limb segments, their arrangement, the configuration of the segments in space, and the shape of the body surface [10], playing an important role, as a classical example, in the integration and use of tools by humans [11].

With this multitude of relevant aspects regarding the definition of body schema, it is important to address the lack of a consistent, comprehensive definition. In order to do that, we will understand how the body schema concept is defined, what are the main theories of body schema and discuss both in the context of and with respect to body-centered (egocentric) vs external (exocentric) frames of reference.

\section{Statement of the problem}

The representation of one's own body is quite different from any other type of representation. It arises from the continuous and constant update of bottom-up and top-down information, both from internal and external bodily inputs. These inputs encompass different sources of information (e.g. visual, proprioceptive, interoception, nociception, motor behavior, etc.) and their interaction allows for building up body representations. Due to the complexity of such representation, it is widely accepted that there is more than one single body representation and many studies have tried to functionally fractionate different body representations, although, up to now, there is not a universally accepted taxonomy [1].

The identification of components of body representation is a challenge and the main interest in studying the egocentric frame of reference relies on the necessity of better understanding the firstperson experience [2]. 
The lack of a proper definition of body schema has been leading to enduring methodological and conceptual misunderstandings in various fields. In rehabilitation, this lack of definition, as well as the unclear contribution of the concept of body schema in the context of the different frames of reference, ego- and exocentric, create a gap in the translation of this knowledge in the context of improving patient care. This happens mostly by the lack of proper integration of somatic and sensory impairment into more comprehensive and inclusive rehabilitation strategies.

\section{Definitions of body schema}

Intrinsic spatial abilities are what allow individuals to navigate the world around them, such as when they manipulate an object, which can be familiar to them or not, or when they play an accurate sports gesture, flourishing from hours and hours of intense training. Tolman was the first to bring the idea that individuals use "cognitive maps" to represent spatial information and navigate in the environment. Specifically, it is traditionally accepted that the distinction between two types of "frames" used to represent spatial information: The egocentric frame, which includes spatial information about the location of the individual in the environment, and the allocentric (or exocentric) frame, which involves the spatial information about the position of objects relative to each other in the surrounding environment [12].

From the beginning of the discussion on body schema, Head defined it as a postural model of the body that actively organizes and modifies the impressions produced by incoming sensory impulses in such a way that the final sensation of body position, or of locality, rises into consciousness charged with a relation to something that has happened before [9].

However, this definition was mainly focused on the functional distinction between "body schema", that is a sensorimotor representation based on afferent and efferent information that guides actions, and "body image", that is a pictorial description of the body mainly based on visual exteroception, i.e., a more aesthetic point of view of the body. Although there was an attempt of differentiating body image in more fine grained definitions, such as "visuospatial body map", which consists of a structural description of the relationships between body parts (also called "body structural description" or "topological map of the body"), and a "body semantics", which corresponds to a conceptual and linguistic body representation, all taxonomies seem to agree with the importance of functional differentiations among different types of body repre- sentation, especially for what attains the role of guiding action for the body schema.

The definition of the body schema has many variations depending on the field of research wherefrom the definition arises. As an example, in the studies related to anorexia nervosa, the body schema definition is defined as "an unconscious, sensorimotor, representation of the body that is invoked in action". However, body schema is not only unconscious or only invoked in action. It is also used for offline motor simulation, including conscious motor simulation (motor imagery) [13].

There is a consensus among authors on the importance of the sensory information. However, they define body schema differently as is listed below. Body schema can be defined as a continuously integrated and updated by proprioceptive, vestibular and motor signals in order to maintain accurate spatial representations [14]. Some simplify, defining it as a sensorimotor representation based on afferent and efferent information that guides actions [1].

A few authors use a more general (superficial) approach, defining body schema as sensorimotor representations of the body that guide actions without awareness or the necessity of conscious monitoring [15], or internal spatial and biomechanical representations of the body, constructed from "on-line" multisensory integration [16].

In an attempt to scrutinize intrinsic aspects of it, some authors relegate body schema to motor control and simulation, adding terms such as body percept (the mental image we have of our bodies) and a representation so-called tactile form (used for certain kinds of tactile perception) [13], constantly minimizing the definition of body schema by mentioning briefly in the middle of a sentence, stating that body schema is comprised of neural representations of body size and position [17], that it is an online representation of body position in space [18] or too ordinarily put, the current limb posture [19].

In addition, some authors understand the concept of body schema by considering only the motor aspects of it, ignoring the sensory aspect of it, defining body schema as intended motor actions that have an impact on receptive fields and spatial representation in the CNS [20] or as the dynamic representation of intrinsic spatial relations of the body [21]. Some appeal to approximating the definition to the concept of postural organization and use the term 
"dynamic postural model", stating that this so-called model would serve as an "egocentric reference" and would correspond to the concept of "body schema", which can be dated back to the beginning of the twentieth century $[9,22]$.

This highly variable set of definitions can be an issue because it brings additional inputs to what is already non-standardized and not clearly well-known. As an example, Haggard and Wolpert brought up the idea that the best word to be used is "scheme" instead of schema [10]. This was deemed to be non-fruitful and only caused more noise amid this discussion. Therefore, there is still the necessity of a clear definition (and terminology) and a better understanding of the relation between body schema and the ego- and exocentric frames of reference.

Some features of body schema are described in the literature and will help guide the search for a better definition. Seven features were summarized and described by Haggard and Wolpert in order to provide a better understanding of body schema, which according to them is a) Spatially coded: affirming that the body schema represents position and configuration of the body in space; b) Modular: postures might be stored as individual entries in a database and the brain represents different body parts in different neural modules, using a modular network to represent all postures; c) Updated with movement: body representation used for action is continuously tracked and updated as we move; d) Adaptable: the body schema must adapt to changes in the spatial properties of the body; e) Supramodal: The body schema receives multiple sensory inputs and integrates them to describe the body as an object in external space; f) Coherent: the brain maintains a coherent spatial organization of the body schema across space and time, ensuring a continuity of body experience, adjusting for biases arising from the use of multiple sensory systems; and g) Interpersonal: a common body schema is used to represent both one's own body, and the bodies of others, facilitating the integration and coping with other's gestures and activities [10].

These features will certainly contribute to the better understanding and definition of body schema. However, before this discussion it is necessary to look at the different frames of reference that relate to the study of body schema and the main theories of body schema.

\section{Frames of reference}

Two major frames of reference are described in literature, the egocentric frame of reference, or so-called body-centered, that uses a proprioceptive, self-referenced coordinate system; and, the exocentric, also called external frame of reference or object-centred, which uses externally-referenced map of visuomotor space to determine the reaching path towards the object [4].

The egocentric frame of reference is based on subject-to-object relations and leads to the creation of body-centered representations (self-centered representations) [14]. An egocentric frame is fundamental in visuomotor control, as the planning and execution of an action needs the representation of the target location in relation to the body. Differently, the egocentric frame is supposedly acquired later in life and is founded on world-based coordinates; within this reference, locations are described using object-to-object relationships, independently from the subject's point of view (world-centered representations) [14,23]. It has been a while since there is a profuse number of papers investigating the relationship between egocentric and egocentric frames of reference, trying to explain how these processes combine to provide healthy and efficient spatial abilities [7]. Generally, most authors agree that both frames allow for the development of spatial representations necessary to accomplish navigation. Successful navigation does not rely on one single frame, but requires the ability to switch and combine different spatial strategies flexibly, depending on the environmental requirements. Interestingly, a great variability has been observed, as individuals may differ in the way they preferentially use egocentric or allocentric strategies [24].

Neuroimaging studies have found that the way we perceive others affect our motor performance and that different areas of the cortex, such as the posterior parietal cortex (PPC) are specialized for egocentric and non-egocentric perspectives [25]. Indeed, the parietal cortex plays a crucial role in the processes linking sensation to action, integration of visual and somatic inputs. This is required for the generation of representations of posture and movement and their spatial relation to external stimuli so that not only the location of an object in space, but also of the potential actions on it, are coded. These converging signals co-vary depending on head position and body orientation as well as auditory and vestibular inputs. Accordingly, the PPC may generate multiple simultaneous representations in eye-, head-, body-, and world-centered frameworks, rather than a single reference for spatial localization [26].

To identify the movement of a specific body part, the brain constructs a representation of the general body structure. In doing this, the brain relies on the so-called body schema, which encom- 
passes the perceptions and dynamics of an individual's own body in relation to that of another. Neuroimaging evidence suggests that a multimodal representation of one's own body might be involved in the perception of other people's bodies [25].

\section{Theories of body schema}

One of the most classic attempts to describe a "representation" of the way the body schema is reflected in the brain comes from the work of Wilder Penfield and his associates. It is a very popular illustration, mainly due to the demonstration on how the body parts are implemented in the brain through the use of an anthropomorphic image of the so called "sensory and motor homunculus" (Figure 1) [27]. However, after decades of discussion and further interpretations of this concept, in the later paper published in 1950 [28], Penfield and Rasmussen added that "although there is usually a close correspondence between somatic sensory and motor representations, the correspondence is not complete. It must be remembered that the representation of sensation refers to specific areas and parts while the motor representation refers to movements of those parts. Different movements of the same part may have different localizations" (Penfield and Rasmussen, 1950, p.215) [29].
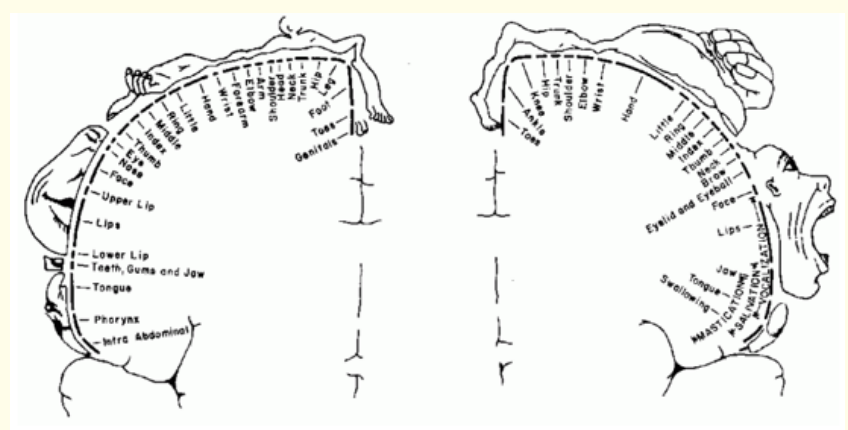

Figure 1: Original diagram of the sensory and motor homunculi (Penfield and Rasmussen, 1950).

It is already known that proprioception functions even before birth, and the observance of certain imitation gestures in a neonate, e.g., facial gestures, suggests that although limited, healthy infants are born with good motor control [29]. This is not different with the body schema and research suggests that the development of body schema begins prenatally, as the fetus experiences vestibular, proprioceptive, and tactile stimuli mainly due to constant tactile input in the containment of the uterus, strong vestibular-proprioceptive stimulation such as the floating in the amniotic fluid, as well as the active fetal movements, that accompanied by the movements of the mother, have a potential influence on body schema development. In addition, processing these sensory inputs is believed to be relevant for later perceptual and cognitive development [30].

Following with the idea of an innate body schema, developed prenatally, Melzack affirms that "there is a convincing evidence that the neonatal brain is genetically programmed to develop neural networks that subserve the perception of the body" (Melzack., et al. 1997, p. 1603) stating that a) there is a neural representation, or network, of the body that, when active, gives rise to perceptual experiences of our body; b) the neural representation in question is distributed in the sensory cortex and the areas throughout parietal lobes; and c) this so-called neuromatrix is, in part, innately determined [31].

The development of body schema continues postnatally and some authors emphasize the theory that sensory impulses are the origin of body schema as the appreciation of movement itself is essential for the body schema development, e.g., when children perform purposeful movement in a certain environment, having the opportunity to develop and understand the many unfolding results about their bodies, thus using simultaneous, multiple sensory sources (e.g., tactile, proprioceptive, visual, and vestibular). In this scenario they begin to develop awareness on the relationship of various body parts and to distinguish between their own bodies and the bodies of others $[32,33]$.

In addition, in the postnatally development of body schema, the somatosensory input is important for development of the body schema as well as for perception of the orientation of the body in space. Active movements of the body also contribute to the development of the body schema through many different factors, such as active exploration of the body's boundaries and contours when one learns the difference between self and non-self, what their body parts are, how they can control them, and what movements of the body feel like. Furthermore, practice and feedback from movement help refine the definition and development of the body schema, building a better body boundary that is made more distinct and specialized [34,35].

As the development and enhancement of body schema is timeand experience dependent, the effect of aging on body schema is also mentioned in aging studies. Spatial abilities and exo /egocentric computations undergo a physiological decline through life as a 
reflex of the related physiological changes of the aging brain. These neurobiological changes, such as decreasing white matter integrity and grey matter volume, have been found in post-mortem and neuroimaging studies revealing important age-related neurobiological changes where, in addition to these changes, associative cortices are more vulnerable to aging deterioration [14]. Since the body schema is known to rise from a multiple systems fine-tuned relationship, even for the representing the location of tactile stimuli in space [36], when these associative cortices are deteriorated, the result is a change in body schema.

Indeed, older adults often report reduced spatial skills, with important consequences on quality of life, safety and autonomy. These spatial impairments can have negative repercussions, as the elderly may avoid navigating and exploring new environments [37], which in turn may further affect their lifestyle [14]. This is an important factor for rehabilitation professionals when designing a treatment plan for elderly and/or patients with neurological impairments.

In addition to the prenatal, postnatal and aging theories of development and decline of body schema, some authors have identified in the past some aspects of body schema from a psychological point of view. Françoise Dolto, a French pediatrician and psychoanalyst, in another attempt to differentiate body schema from body image, defined body schema as an anatomical entity; a representative of the human species independent of time and space, identical for every human being. According to Dolto, body image though is a particular entity, constituted throughout a subject's subjective history, it is a living synthesis of one's emotional experiences [38]. Nonetheless, this seems to be difficult to reconcile, as Shontz (1969) pointed that because Head, in 1911, proposed the concept of "body schemata" using a physiological perspective, Fisher and Cleveland [39] pointed that this was a problem because this identification makes it impossible to investigate experience and/or behaviour alone [40]. Therefore, the investigation of body schema calls for a mixed-model approach, where both psychological (behavioural) and physiological aspects are considered in order to contribute evidence towards a common answer without relegating it to subjective and/or unilateral methods.

\section{Discussion}

This question focusses on the study of body schema. However, the concept of body image and the differentiation of body schema and body image is necessary, especially when the two concepts are intertwined and used interchangeably, as we could observe from the work of Françoise Dolto [38] previously described. What is relevant to be discussed from Dolto's point of view is that there is a dynamic interaction between body image and body schema as she states that "a pathological body image can disturb the functioning of an intact body schema, and vice versa. The most stunning is the clinical finding that a damaged body schema can go hand in hand with a normal body image, if a child is allowed to play verbally with its body in interaction with significant others" (Preester and Knockaert, 2005, p. 17).

Regarding the relationship of body schema with exo- and egocentric frames of reference, there is an understanding that there is a bi-directionality of this relationship, once the body schema development and refining feeds from the relationship with the environment and the tasks performed in it. As another example, the location of tactile stimulation in the skin can be encoded in both frames of reference (relative to the skin surface or egocentric; and, relative to the position of the stimulus in external space, exocentric) [36]. Interestingly, as the stimuli is encoded in an egocentric frame of reference, coordinates of the body are locked and move with it. Alsmith also points out that this is challenging since the body is not a single point in space but rather a group of moving parts, leading to the suggestion that even within the egocentric frame of reference, each physical part of the body plays a role in determining and updating the egocentric frame of reference, thus adjusting to task demands, e.g., when the movement is anchored in different parts of the body [2]. This goes along with the features of body schema listed by Haggard and Wolpert in 2005, since this fits in the description of a system that is spatially coded, modular, adaptable, coherent, and more importantly in this example, updated with movement [10]. Alsmith also highlights the importance of the head and the torso as major factors in specifying the egocentric frame of reference. Although according to the author there is a lack of definitive evidence, it is suggested that the head is a strong candidate due to the extremely advanced and refined sensory organs, while on the other side, the torso plays an important role in anchoring the limbs and providing a firm, voluminous base where the appendices attach thereto [2].

In an experiment on tool usage and visual receptive field of a monkey, Martel., et al. found that when the monkey used a rake as an extension of its hand there was an enlargement of the monkey's visual receptive field as there was the incorporation of the tool as an extension of the hand. Interestingly, the visual receptive fields 
return to their original size within a few minutes after the tool use is discontinued, and they do not expand at all if the monkey simply holds the rake without intending to use it [41]. This also goes towards the features of body schema previously described and is an indication that body schema, as an internal representation of the body parts, is not only updated with movement and rapidly influenced by sensory inputs (e.g., vision), but is also task-dependent, although more evidence is necessary to confirm this statement. Especially when commenting on the role of sensory perception Haggard and Wolpert imply that interpersonal function necessarily implies a supramodal body schema, since information about others' bodies is generally visual, while information from one's own body is generally tactile or proprioceptive. [10]. The likely presence of multiple representations of the body within the egocentric frame of reference makes it difficult to specify a definitive theoretical definition of body schema and frame of references but rather indicates an even more complex level, like pieces of a puzzle, working as subrepresentations within each of the reference frames (See figure 2 for an illustration of the complex and sparse processing of spatial encoding in the healthy brain).

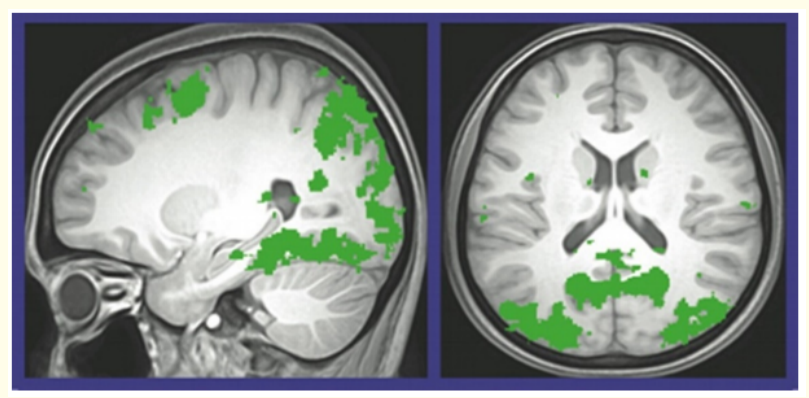

Figure 2: Brain regions identified as active in green in a wholebrain functional analysis for spatial encoding in healthy individuals illustrating the sparse activation across multiple regions of the brain. Adapted from [24].

Another finding that contributes evidence towards a egocentric body schema is the absence of the phantom limb perception in people with congenital limb absence, suggesting that an innate representation is retained and modified throughout life [31] and most likely provide a template (e.g., neuromatrix as mentioned by Preester and Knockaert, 2005) from which phantom limbs are perceived following both congenital and/or acquired limb loss [5].

Aging also plays a role in determining the egocentric frame of reference as a major factor in the development, maintenance and refining of one's body schema. Evidence suggests that there is a relation between aging and spatial frames. This was conducted through a series of cognitive tasks and focusing on different cognitive domains (e.g., like spatial memory, spatial navigation, mental rotation or spatial encoding). These impaired exocentric computations are usually related to hippocampal deterioration. On the other hand, the effect of aging on the egocentric frame is more controversial, especially depending on the considered cognitive domain. Nevertheless, age-related exocentric impairments might as well reflect the decreasing ability to switch from egocentric to allocentric frame of reference, pointing to the direction that indicate the role of executive functions in affecting such abilities [14]. The limitation of the studies and the lack of definitive understanding of these impairments, do not eliminate the potential impact of the study of body schema in rehabilitation research, e.g., investigation related to increasing elderly falling rates, especially when it is known that ability to interact with one's peripersonal space has both a safety and an action value [42].

\section{Conclusion}

Establishing a definition of body schema is still very challenging since there is not definitive evidence about the multiple factors directly influencing it, e.g., the clear understanding on which frame (or frames) of reference wherefrom the body schema would arise. In addition, as commonly observed in many other cases (e.g., coordination) the definition varies between different disciplines and even amid rehabilitation researchers and clinicians. Due to this lack of an accurate definition, there is also an unclear justification for the choice of measures used to quantify it or even determine the extent to which the body schema would be related to sensorimotor impairments, e.g., patients with compromised visual acuity or compromised sensorimotor functions post-stroke.

Based on the above-mentioned definitions and discussion on the main features of body schema, I would like to propose a novel definition for body schema that is more accurate and encompasses its construct that has been long pursued by physical and occupation therapists [30]: Body schema is a spatiotemporal, continuously updated, modular and coherent representation of the physical body in the central nervous system, integrating a multitude of sensorimotor processes, serving as a guide for movements to be performed as accurately as possible while adapting to the constraints of the environment.

In summary, although there is a relative interest of researchers from different fields in the investigation of body schema, there is 
not yet a definitive theoretical framework that can determine the origin and the meaning of body schema. Therefore, future research is necessary to clarify certain aspects involved in the body schema investigation that are relevant to rehabilitation, e.g., the use of deficits in body schema (e.g., agnosia, hemi-neglect, etc.) as indicators of a higher, more intricate relationship between body schema and egocentric frame of reference.

\section{Bibliography}

1. Di Vita A., et al. "To move or not to move, that is the question! Body schema and non-action oriented body representations: An fMRI meta-analytic study". Neuroscience and Biobehavioral Reviews 68 (2016): 37-46.

2. Alsmith AJT., et al. "Dissociating contributions of head and torso to spatial reference frames: The misalignment paradigm". Consciousness and Cognition 53 (2017): 105-114.

3. Paillard J. "Cognitive versus sensori-motor encoding of spatial information". In Cognitive processes and spatial orientation in animal and man. P. Ellen and C. Thinus-Blanc, Editors. Martinus Nijhoff: Dordrecht (1987): 43-77.

4. Paillard J. "Motor and representational framing of space, in Brain and Space". J. Paillard, Editor. Oxford University Press: Oxford. (1991): 163-182.

5. Giummarra MJ., et al. "Mechanisms underlying embodiment, disembodiment and loss of embodiment". Neuroscience and Biobehavioral Reviews 32.1 (2008): 143-160.

6. Swindale N. "Cortical cartography: what's in a map?" Current Biology 11.19 (2001): R764-R767.

7. Avraamides MN and JW Kelly. "Multiple systems of spatial memory and action". Cognitive Process 9.2 (2008): 93-106.

8. Descartes, R., et al. "The philosophical writings of Descartes". 1984, Cambridge [Cambridgeshire]; New York: Cambridge University Press (1984).

9. Head H and G Holmes. "Sensory disturbances from cerebral lesions". Brain 34.2-3 (1911): 102.

10. Haggard P and DM Wolpert. "Disorders of body scheme". Higher-order motor disorders. ed. H.-J. Freund., et al. Oxford: Oxford University Press (2005).

11. Macaluso E and A Maravita. "The representation of space near the body through touch and vision". Neuropsychologia 48.3 (2010): 782-795.
12. Tolman EC. "Cognitive maps in rats and men". The Psychological Review 55.4 (1948): 19.

13. Gadsby S. "Distorted body representations in anorexia nervosa". Consciousness and Cognition 51 (2017): 17-33.

14. Colombo, D., et al. "Egocentric and allocentric spatial reference frames in aging: A systematic review". Neuroscience and Biobehavioral Reviews 80 (2017): 605-621.

15. Gallagher S. "Body Image and Body Schema: A Conceptual Clarification”. 7 (1985): 541-554.

16. Falconer CJ and FW Mast. "Balancing the mind: vestibular induced facilitation of egocentric mental transformations". EXperimental Psychology 59.6 (2012): 332-339.

17. Kabasakalian, A., et al. "Hypometric allocentric and egocentric distance estimates in parkinson disease". Cognitive and Behavioral Neurology 26.3 (2013): 133-139.

18. Medina J and C DePasquale. "Influence of the body schema on mirror-touch synesthesia”. Cortex 88 (2017): 53-65.

19. Ali JB., et al. "Effects of posture on tactile localization by 4 years of age are modulated by sight of the hands: evidence for an early acquired external spatial frame of reference for touch". Developmental Science 17.6 (2014): 935-943.

20. Colby CLC. "Action-oriented spatial reference frames in cortex". Neuron 20.1 (1998): 15-24.

21. Creem-Regehr SH., et al. "Neural correlates of two imagined egocentric transformations". NeuroImage 35.2 (2007): 916927.

22. Galati G., et al. "Multiple reference frames used by the human brain for spatial perception and memory". Experimental Brain Research 206.2 (2010): 109-120.

23. Milner AD and MA Goodale. "Visual pathways to perception and action". Progress in Brain Research 95 (1993): 317-37.

24. Marchette SA., et al. "Cognitive Mappers to Creatures of Habit: Differential Engagement of Place and Response Learning Mechanisms Predicts Human Navigational Behavior". The Journal of Neuroscience 31.43 (2011): 15264-15268.

25. Bruzzo A., et al. "Hand-object interaction in perspective". Neuroscience Letters 441.1 (2008): 61-65.

26. Binkofski F., et al. "Mirror agnosia and mirror ataxia constitute different parietal lobe disorders". Annals of Neurology 46.1 (1999): 51-61. 
27. Penfield W and E Boldrey. "Somatic motor and sensory representation in the cerebral cortex of man as studied by electrical stimulation". Brain 60.4 (1937): 389-443.

28. Penfield W and T Rasmussen. "The cerebral cortex of man: A clinical study of localization of function". Journal of the American Medical Association 144.16 (1950): 1412-1412.

29. Preester Hd and V Knockaert. "Body image and body schema: interdisciplinary perspectives on the body". Amsterdam; Philadelphia: J. Benjamins (2005).

30. Mitchell AW. "Theories of Body Scheme Development. Physical and Occupational Therapy". Pediatrics 17.4 (1998): 25-45.

31. Melzack R., et al. "Phantom limbs in people with congenital limb deficiency or amputation in early childhood". Brain 120 (1997): 1603-1620.

32. Schilder P. "The image and appearance of the human body: Studies in the constructive energies of the psyche". A.M.A. Archives of Neurology and Psychiatry 66.1 (1951): 116-117.

33. Ayres AJ. "Development of the body scheme in children". American Journal of Occupational Therapy 15 (1961): 99-102.

34. Lewis M and J. "Brooks-Gunn, Social cognition and the acquisition of self”. New York: Plenum Press (1979).

35. van der Velde CD. "Body images of one's self and of others: developmental and clinical significance". The American Journal of Psychiatry 142.5 (1985): 527-537.

36. Medina J and HB Coslett. "From maps to form to space: Touch and the body schema”. Neuropsychologia 48.3 (2010): 645654.

37. Burns PC. "Navigation and the Mobility of Older Drivers". The Journals of Gerontology: Series B 54B.1 (1999): S49-S55.

38. Hall G., et al. "Theory and practice in child psychoanalysis: an introduction to the work of Françoise Dolto" (2009): 224.

39. Fisher S and S Cleveland. "Development and Structure of the Body Image”. Ed. T. Francis. Psychology press (2014): 344.

40. Shontz FC. "Perceptual and cognitive aspects of body experience". New York: Academic Press (1969).

41. Martel M., et al. "Tool-use: An open window into body representation and its plasticity". Cognitive Neuropsychology 33.1-2 (2016): 82-101.
42. Coello Y., et al. "Embodied perception of reachable space: how do we manage threatening objects?" Cognitive Processing 13.1 (2012): 131-135.

\section{Assets from publication with us}

- Prompt Acknowledgement after receiving the article

- Thorough Double blinded peer review

- Rapid Publication

- Issue of Publication Certificate

- High visibility of your Published work

Website: www.actascientific.com/

Submit Article: www.actascientific.com/submission.php

Email us: editor@actascientific.com

Contact us: +919182824667 